\title{
Close binary system GO Cyg
}

\author{
B. Ulaş ${ }^{\text {a,* }}$, B. Kalomeni ${ }^{\text {a,b }}$, V. Keskin ${ }^{\text {a }}$, O. Köse ${ }^{\text {a }}$, K. Yakut ${ }^{\text {a,c,1 }}$ \\ ${ }^{a}$ Department of Astronomy and Space Sciences, University of Ege, 35100 Bornova-İzmir, Turkey \\ ${ }^{\mathrm{b}}$ Department of Physics, Izmir Institute of Technology, Turkey \\ ${ }^{\mathrm{c}}$ Institute of Astronomy, University of Cambridge, Madingley Road, Cambridge CB3 OHA, UK
}

\section{A R T I C L E I N F O}

\section{Article history:}

Received 20 July 2011

Received in revised form 6 September 2011

Accepted 7 September 2011

Available online 29 September 2011

Communicated by P.S. Conti

\section{Keywords:}

Stars: binaries: eclipsing

Stars: binaries: close

Stars: binaries: general

Stars: fundamental parameters

Stars: low-mass

\begin{abstract}
A B S T R A C T
In this study, we present long term photometric variations of the close binary system GO Cyg. Modelling of the system shows that the primary is filling Roche lobe and the secondary of the system is almost filling its Roche lobe. The physical parameters of the system are $M_{1}=3.0 \pm 0.2 M_{\odot}, M_{2}=1.3 \pm 0.1 M_{\odot}$, $R_{1}=2.50 \pm 0.12 R_{\odot}, R_{2}=1.75 \pm 0.09 R_{\odot}, L_{1}=64 \pm 9 L_{\odot}, L_{2}=4.9 \pm 0.7 L_{\odot}$, and $a=5.5 \pm 0.3 R_{\odot}$. Our results show that GO Cyg is the most massive system near contact binary (NCB). Analysis of times of the minima shows a sinusoidal variation with a period of $92.3 \pm 0.5 \mathrm{yr}$ due to a third body whose mass is less than $2.3 M_{\odot}$ Finally a period variation rate of $-1.4 \times 10^{-9} \mathrm{~d} / \mathrm{yr}$ has been determined using all available light curves.
\end{abstract} (c) 2011 Elsevier B.V. All rights reserved.

\section{Introduction}

Studies of the evolution of late-type close binary systems reveal that the evolution of detached, semi-detached and contact systems are closely related (Yakut and Eggleton, 2005; Eggleton, 2010 and reference therein). The more massive star in a detached binary system fills its Roche lobe first because it has shorter evolutionary timescale before its companion. The system is semi-detached binary. In addition to nuclear evolution and mass loss, mass transfer has a crucial role in driving a binary towards a contact phase of evolution. The observations of detached, contact and semi-detached binaries are crucial to our further understanding of the evolution of close binary systems.

We therefore, include GO Cyg (HD 196628, GSC 02694-00550, $V=8^{m} \cdot 47$, A0V) into our close binary stars observation programme (see Ulaş et al., 2011; Köse et al., 2011). The system is a $\beta$-Lyr type (short period $0^{d} \cdot 71$ ) binary system and observations of the binary cover eighty years. Following its discovery by Schneller (1928) the system has been extensively studied by many authors. (Payne-Gaposchkin, 1935; Pierce, 1939; Popper, 1957). Ovenden (1954), Mannino (1963), Rovithis et al. (1990), Sezer et al. (1993), Jassur (1997), Rovithis-Livaniou et al. (1997), Edalati and Atighi (1997), Oh et al. (2000), Zabihinpoor et al. (2006) studied the system photometrically. Using different methods in analysis most

\footnotetext{
* Corresponding author.

E-mail address: bulash@gmail.com (B. Ulas).

1 Visiting astronomer during the summer of 2011.
}

studies agree with the primary filling its Roche lobe. Asymmetry in the secondary minimum have been discussed in previous studies (e.g. Edalati and Atighi, 1997; Zabihinpoor et al., 2006). Pearce (1933) found the mass function and mass ratio of 0.85 for GO Cyg. Later studies have reported higher mass ratios. Pribulla et al. (2009) examined the binary and classified it as a member of a group called difficult binary stars. In this group accurate radial velocities are not available. Pribulla et al. (2009) concluded that the temperature difference between the components makes the system a difficult candidate in determination of the ideal broadening function. A velocity value of $v \approx 35 \mathrm{~km} / \mathrm{s}$ for a third body was given in Pribulla et al. (2009).

Period variation of GO Cyg has been studied by many investigators. Period increase was discussed in a number of studies (e.g. Sezer et al., 1985; Rovithis-Livaniou et al., 1997; Edalati and Atighi, 1997; Zabihinpoor et al., 2006). Jones et al. (1994) reported a sinusoidal variation superimposed on a parabolic trend. Elkhateeb (2005) noted a period increase of $d P / d t=1.28 \times 10^{-7}$ which is close to that of Oh et al.'s (2000) value of $1.51 \times 10^{-7}$. Hall and Louth (1990) discussed a magnetic cycle by studying the period decrease between the year 1934 and 1984. Chochol et al. (2006) represented the $O-C$ curve by a sinusoidal fit by using the Cracow database and their data. A third body with an orbital period of $90 \mathrm{yr}$ and a mass of $0.62 M_{\odot}$ has been proposed.

In the following sections, we present our new observations of GO Cyg. We preformed photometric analysis, period variations and compare our results with its previously published works. All the available light curves were collected from the literature and 
studied for various physical processes (e.g. magnetic activity, mass transfer, third light) and their variations. The $O-C$ variation with recently obtained times of minima revealed the discrepancy between the results of the light curve solution and period study of earlier studies. In this study, therefore, we investigate different possibilities that cause period variation in order to reveal the most accurate structure and behavior of the components. The physical parameters of the system are given with a discussion on the evolutionary status of the binary.

\section{New observations}

The light variation and minima times of GO Cyg obtained in the Bessel $B, V$, and $R$ bands in 16 nights between June - August 2007 and one night in April 2011. The observations carried out at TÜBITAK National Observatory (TUG) and Ege University Observatory with the $40 \mathrm{~cm}$ telescope using an Apogee CCD U47. Comparison and check stars are selected as GSC 02694-00280 and GSC 02694-00733, respectively. The total number of the points obtained during the observations are 3715 in $B, 3726$ in $V$, and 3698 in $R$ band. IRAF (DIGIPHOT/APPHOT) packages are used in data reduction. Standard deviations of the data are estimated as $0^{\mathrm{m}} \cdot 04,0^{\mathrm{m}} \cdot 017$, and $0^{\mathrm{m}} \cdot 015$ for $B, V$, and $R$ bands, respectively.

In Fig. 1 we show the $B, V$, and $R$ light curves of GO Cyg. In this study, we do not find the apparent asymmetry in $0.6-0.7$ orbital phase previously reported by Edalati and Atighi (1997), Zabihinpoor et al. (2006). In data our reduction and analysis, we used the linear ephemeris described by Sezer et al. (1993).

\section{Eclipse timings and period study}

Cester et al. (1979) reported an orbital increase of $Q=0.7 \times$ $10^{-10}$ based on seven nights observational data obtained between 1972-1975. Sezer et al. (1985) also reported an increase with a $Q$ value of $1.13 \times 10^{-10}$ days. Hall and Louth (1990) consider the $O_{-}$ $C$ curve and split it in three region. The first and the third region

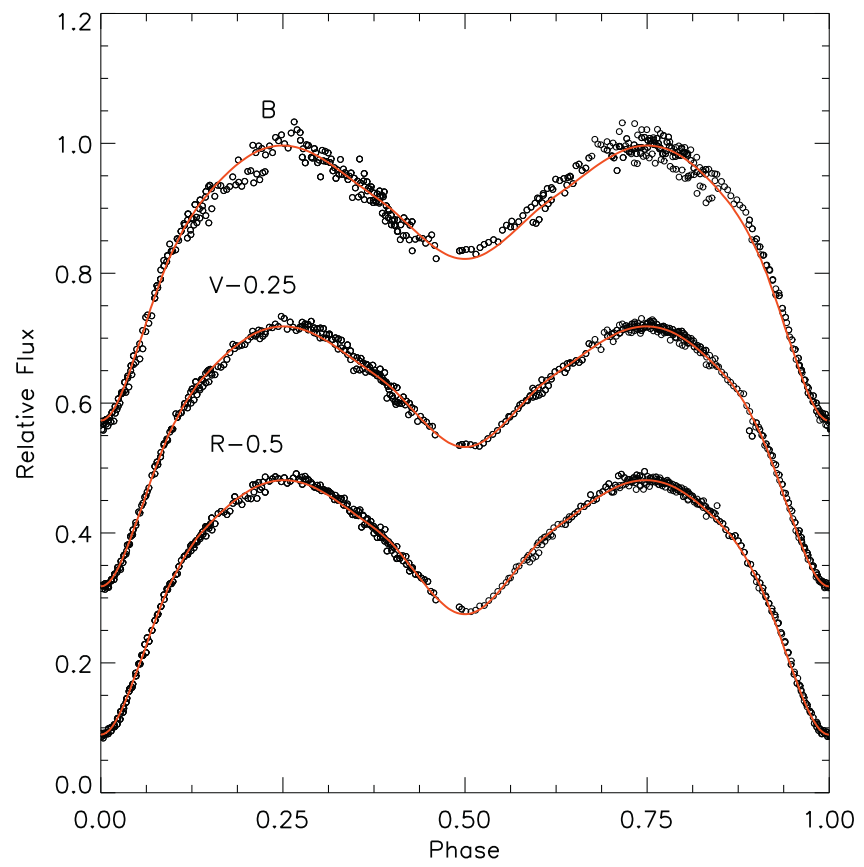

Fig. 1. The observed and the computed (solid line) light curves of the system GO Cyg. The light curves in $V$ and $R$ bands are moved by a value of 0.25 and 0.5 , respectively, for the sake of comparison. of the curve showed sudden variation. Therefore the authors analysed these regions under linear assumption while the second part was presented by a quadratic fit with a period increase of $Q=$ $1.28 \times 10^{-10}$. The authors concluded that this behavior of the $O-$ $C$ curve can be attributed to a magnetic cycle. Jones et al. (1994) showed that the residuals of the parabolic fit show a sine-like variation with a period of $38.9 \mathrm{yr}$. A period increase was also discussed by Rovithis-Livaniou et al. (1997). A period with $Q=1.6 \times 10^{-10}$ was noted by Edalati and Atighi (1997). Oh et al. (2000) also represented the $O-C$ curve by an upward parabola with $Q=1.47 \times 10^{-10}$. Elkhateeb (2005) found that the period is increasing with a value of $Q=1.26 \times 10^{-10}$. The parabolic and third order polynomial fits
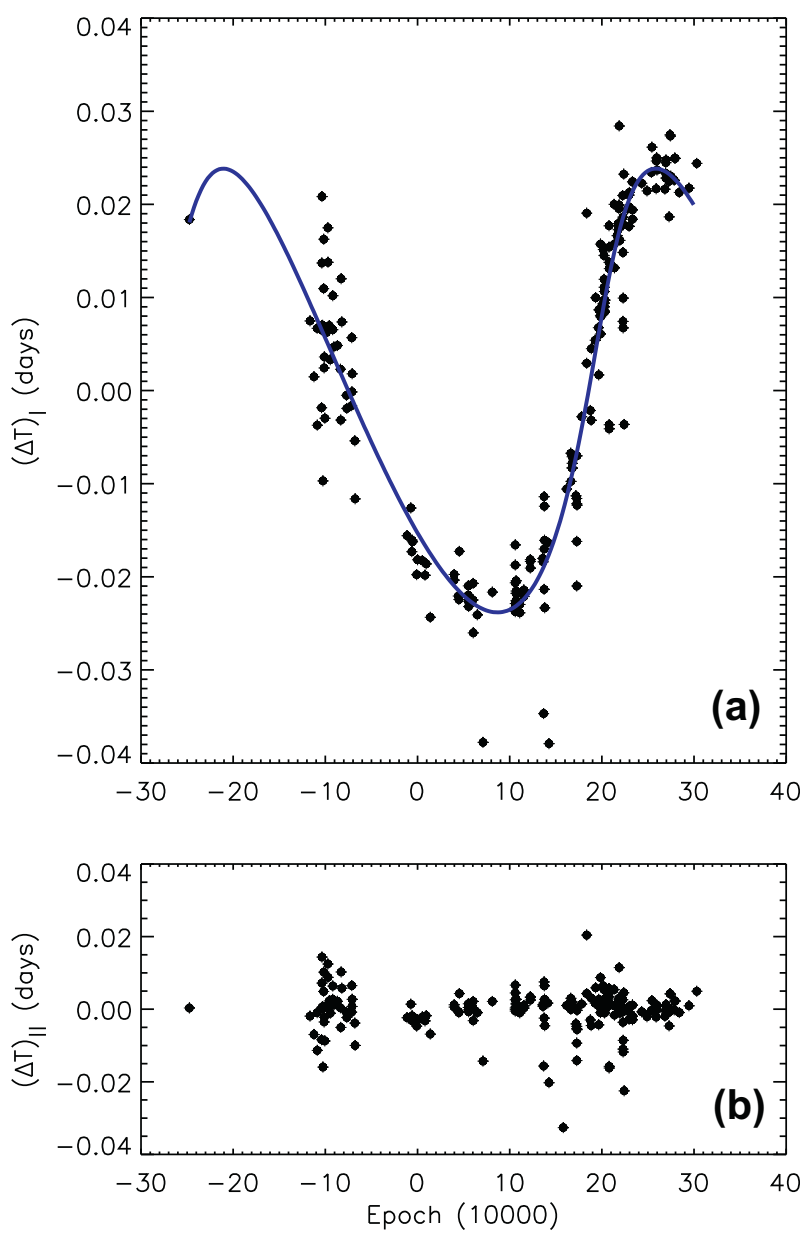

Fig. 2. (a) Residuals for the times of minimum light of GO Cyg. The solid line is obtained with the assumption of sine-like variation. (b) The difference between the observations and the computed sinusoidal curve.

Table 1

Results of the period analysis and the orbital elements of the third body. The standard errors, $1 \sigma$, are given in parentheses.

\begin{tabular}{lll}
\hline Parameter & Unit & Value \\
\hline$T_{o}$ & {$[\mathrm{HJD}]$} & $2433930.4283(7)$ \\
$P_{o}$ & [day] & $0.717764585(15)$ \\
$P^{\prime}$ & [year] & $92.3(5)$ \\
$T^{\prime}$ & {$[\mathrm{HJD}]$} & $2414756(300)$ \\
$e^{\prime}$ & & $0.46(1)$ \\
$\omega^{\prime}$ & {$\left[{ }^{\circ}\right]$} & $20.3(2.2)$ \\
$a_{12} \sin i^{\prime}$ & {$[\mathrm{AU}]$} & $4.57(4)$ \\
$f(m)$ & {$\left[M_{\odot}\right]$} & $0.0112(5)$ \\
$m_{3 ; i^{\prime}=20^{\circ}}$ & {$\left[M_{\odot}\right]$} & 2.30 \\
$m_{3 ; i^{\prime}=90^{\circ}}$ & {$\left[M_{\odot}\right]$} & 0.65 \\
\hline
\end{tabular}


were compared by Zabihinpoor et al. (2006). The authors give the quadratic term as $0.935 \times 10^{-10}$. Zabihinpoor et al. (2006) also

Table 2

The photometric elements and their formal $1 \sigma$ errors of GO Cyg. See text for details.

\begin{tabular}{ll}
\hline Parameter & Value \\
\hline Geometric parameters & $75.67(3)$ \\
$i\left({ }^{\circ}\right)$ & 2.735 \\
$\Omega_{1}$ & $2.686(4)$ \\
$\Omega_{2}$ & $0.428(9)$ \\
$q$ & $0.4542(2)$ \\
Fractional radius of primary & $0.3189(12)$ \\
Fractional radius of secondary & \\
Radiative parameters & 10350 \\
$T_{1}($ K) & $6490(90)$ \\
$T_{2}($ K) & 1.0 \\
Albedo $A_{1}$ & 0.760 \\
Albedo $A_{2}$ & 1.0 \\
Gravity brightening $g_{1}$ & 0.32 \\
Gravity brightening $g_{2}$ & \\
Limb darkening $x_{1}, x_{2}$ & 0.693 \\
$x_{1} B$ & 0.812 \\
$x_{2} B$ & 0.593 \\
$x_{1} V$ & 0.721 \\
$x_{2} V$ & 0.484 \\
$x_{1} R$ & 0.628 \\
$x_{2} R$ & \\
Luminosity ratio: $\frac{L_{1}}{L_{1}+L_{2}+l_{3}}(\%)$ & $94(5)$ \\
$B$ & $90(4)$ \\
$V$ & \\
$R$ & \\
Luminosity ratio: $\frac{l_{3}}{L_{1}+L_{2}+l_{3}}(\%)$ & 0.39 \\
$B$ & \\
$V$ & \\
\hline & \\
\hline & \\
&
\end{tabular}

discussed the inconsistency between geometric configuration and period variation rate. Recently, Chochol et al. (2006) suggested the light time effect for the period variation. The authors discussed that in a binary system where the primary fills its Roche lobe and loses mass a decrease in orbital period can be expected.

Recently we obtained two times of minima $2454318.54864 \pm$ 0.00013 , and $2455676.56220 \pm 0.00016$. The times of minima obtained in this study show that the $O-C$ curve changes shape from early-assumed upward parabola to a sinusoidal variation that supports the previous discussion about a third body in the system. The period variation is studied using a total of 194 data points obtained by photometric/CCD observations. The times of minima are obtained from the literature (Kreiner et al., 2001; Erkan et al., 2010) and those yielded by this study. The weighted least-squares method is used in order to determine the orbital elements of the third body. Sinusoidal variation in the $O-C$ curve, where both the primary and the secondary minima follow the same trend suggests a light-time effect because of the presence of a third component that can be represented by following formula (Irwin, 1959; Kalomeni et al., 2007):

$\operatorname{MinI}=T_{o}+P_{0} E++\frac{a_{12} \sin i^{\prime}}{c}\left[\frac{1-e^{\prime 2}}{1+e^{\prime} \cos v^{\prime}}\left(v^{\prime}+\omega^{\prime}\right)+e^{\prime} \sin \omega^{\prime}\right]$

where $T_{\mathrm{o}}$ is the starting epoch for the primary minimum, $E$ is the integer eclipse cycle number, $P_{\mathrm{o}}$ is the orbital period of the eclipsing binary $a_{12}, i^{\prime}, e^{\prime}$, and $\omega^{\prime}$ are the semi-major axis, inclination, eccentricity, and the longitude of the periastron of eclipsing binary about the third body, and $v$ denotes the true anomaly of the position of the center of mass. Time of periastron passage $T^{\prime}$ and orbital period $P^{\prime}$ are the unknown parameters in Eq. (1).

Our result from our analysis are shown in Fig. 2. Fig. 2a shows the consistency between the observational and model prediction

Table 3

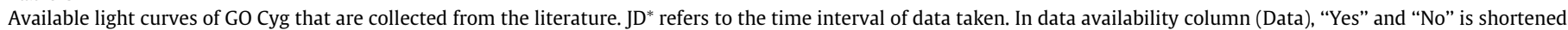

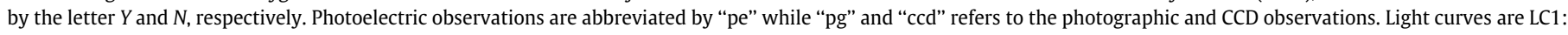

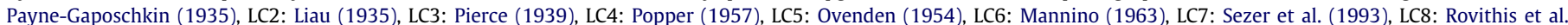

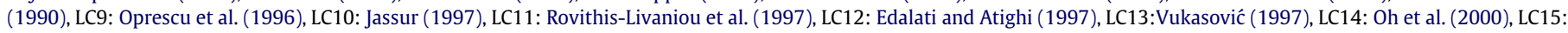
Zabihinpoor et al. (2006), LC16: This study.

\begin{tabular}{|c|c|c|c|c|c|c|c|}
\hline ID & Year & $\mathrm{JD}^{*}(2400000+)$ & Filters & Type & Comparison (s) & $N_{\text {points }}$ & Data \\
\hline LC1 & 1934 & & blue, red & pg & $\begin{array}{l}\mathrm{BD}+364150 \\
\mathrm{BD}+344098 \\
\mathrm{BD}+354197\end{array}$ & & $\mathrm{~N}$ \\
\hline LC2 & 1935 & & & & & & $\mathrm{~N}$ \\
\hline LC3 & 1936 & & $\mathrm{~V}$ & vis & BD+35 4188 & 122 & $\mathrm{Y}$ \\
\hline LC4 & 1950 & $33478.8-33498.0$ & $\mathrm{~B}, \mathrm{~V}$ & pe & HD 196771 & $\mathrm{~B}: 261, \mathrm{~V}: 261$ & $\mathrm{Y}$ \\
\hline LC5 & $1950-51$ & & B, V & pe & $\begin{array}{l}\mathrm{BD}+354197 \\
\mathrm{BD}+344098\end{array}$ & $\mathrm{~B}: 64, \mathrm{~V}: 68$ & $\mathrm{Y}$ \\
\hline LC6 & $1959-62$ & $36782.4-37910.5$ & $\mathrm{~B}, \mathrm{~V}$ & pe & $\begin{array}{l}\mathrm{BD}+354197 \\
\mathrm{BD}+344098\end{array}$ & B:333, V:353 & $\mathrm{Y}$ \\
\hline LC7 & $1984-85$ & $45866.4-46348.3$ & $\mathrm{~B}, \mathrm{~V}$ & pe & $\begin{array}{l}\text { HD } 197292 \\
\text { HD } 197346\end{array}$ & B:416, V:414 & $\mathrm{Y}$ \\
\hline LC8 & 1985 & $46264.3-46329.4$ & $\mathrm{~B}, \mathrm{~V}$ & pe & $\begin{array}{l}\mathrm{BD}+354180 \\
\mathrm{BD}+344098\end{array}$ & B:631, V:633 & $\mathrm{Y}$ \\
\hline LC9 & 1989-92 & & $\mathrm{B}, \mathrm{V}$ & pe & $\begin{array}{l}\mathrm{BD}+354197 \\
\mathrm{BD}+344098\end{array}$ & & $\mathrm{~N}$ \\
\hline LC10 & 1992 & & $\mathrm{U}, \mathrm{B}, \mathrm{V}$ & pe & $\begin{array}{l}\mathrm{BD}+354180 \\
\mathrm{BD}+344098\end{array}$ & & $\mathrm{~N}$ \\
\hline LC11 & 1993-94 & & $\mathrm{B}, \mathrm{V}$ & pe & $\begin{array}{l}\mathrm{BD}+354197 \\
\mathrm{BD}+344098\end{array}$ & & $\mathrm{~N}$ \\
\hline LC12 & 1995 & & $\mathrm{U}, \mathrm{B}, \mathrm{V}$ & pe & $\begin{array}{l}B D+354180 \\
B D+344098\end{array}$ & & $\mathrm{~N}$ \\
\hline LC13 & 1996 & & U, B, V & pe & SAO 70314 & & $\mathrm{~N}$ \\
\hline LC14 & 1996 & $50366.0-50436.0$ & $\mathrm{~B}, \mathrm{~V}$ & pe & $\begin{array}{l}B D+354197 \\
B D+344098\end{array}$ & B:398, V:397 & $\mathrm{Y}$ \\
\hline LC15 & 2002 & & $\mathrm{~B}, \mathrm{~V}$ & pe & $\begin{array}{l}\text { HD } 197292 \\
\text { HD } 197346\end{array}$ & B:545, V:521 & $\mathrm{Y}$ \\
\hline LC16 & 2007 & $54361.5-54321.6$ & $\mathrm{~B}, \mathrm{~V}, \mathrm{R}$ & $\mathrm{ccd}$ & $\begin{array}{l}\text { GSC 02694-00280 } \\
\text { GSC 02694-00733 }\end{array}$ & $\begin{array}{l}\text { B:3711, V:3722, } \\
\text { R:3694 }\end{array}$ & $\mathrm{Y}$ \\
\hline
\end{tabular}


in the assumption of a third body. Fig. $2 \mathrm{~b}$ shows the residuals from a Sinusoidal variation. The orbital elements of a third component are listed in Table 1 . It can be clearly seen from the figure that any investigation of any detailed variation in $(\Delta T)_{I I}$ points makes no sense. We have also investigated orbital period variation of the system with a different method from the $O-C$ analysis. We have re-analysed the light curves from the available eighty years and we discussed further in Section 5 below. We conclude that if the $O-C$ variation shows a downward parabola its period that is too long to determine from available times of minima.

\section{Light curve solution}

The light curve of the system has been analysed by numerous researchers. Ovenden (1954) obtained two-colour light curve and solved them by using Russell's method. The author assumed that the primary dominates observed light. This results in a reflection effect that makes difficult to identify the secondary in the spectrum. Asymmetry between maxima discussed as an intrinsic variation. Mannino (1963) solved the photoelectric $B$ and $V$ light curves of the system with the Russell-Merill method. Rovithis et al.

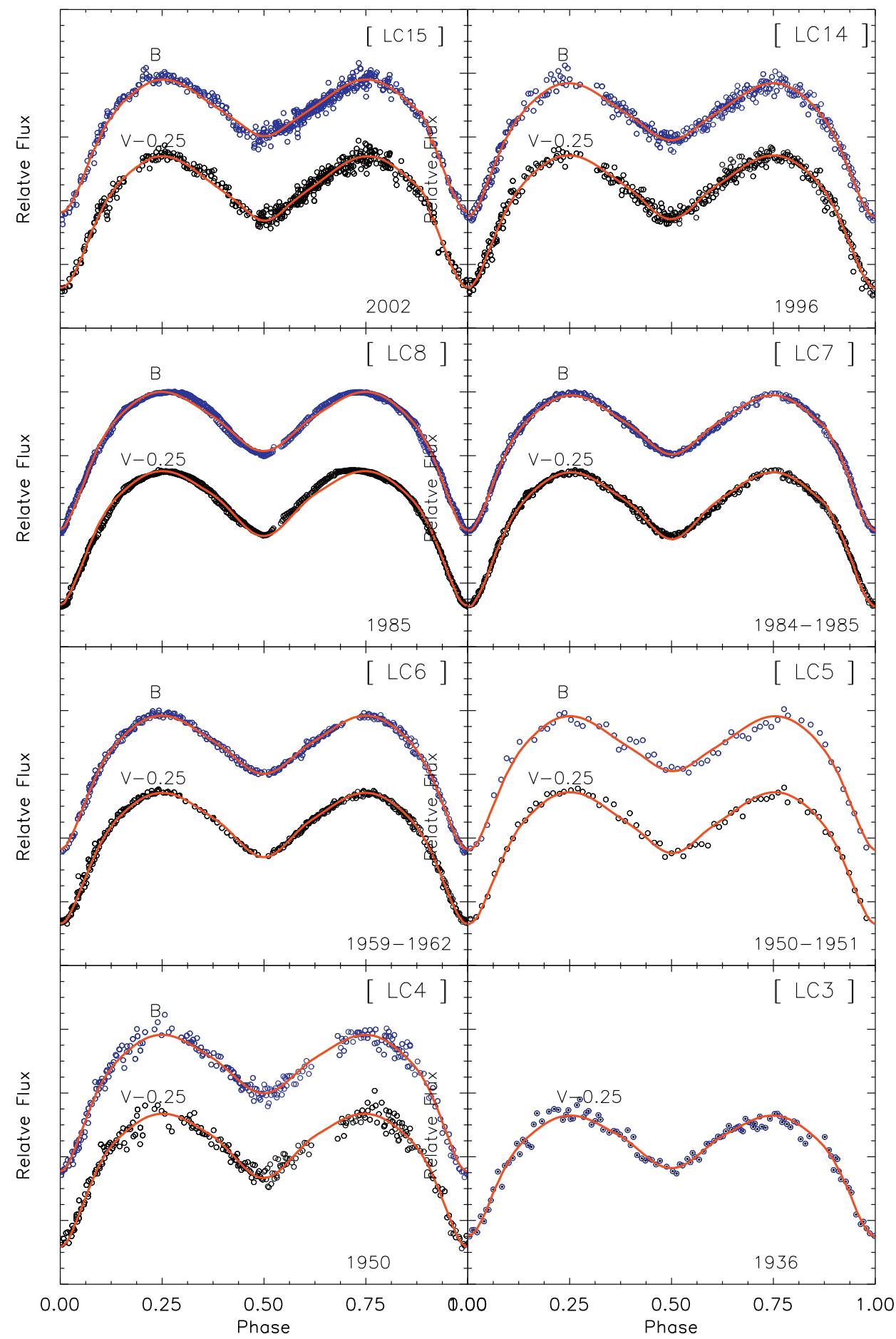

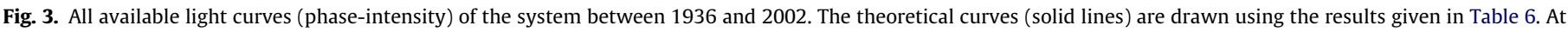
the right bottom of each panel observation years are given. 
(1990) analysed the light curves and estimated the geometric elements by using frequency domain techniques. The authors reported no difference between the level of maxima. The $B V$ light curve combined with Holmgren's radial velocity curve was solved by Sezer et al. (1993) by using Wilson-Devinney (WD) method. The result indicated a semi-detached configuration where the primary is filling its Roche lobe. A standard iterative optimization technique was used by Jassur (1997) to solve the UBV light curves. Rovithis-Livaniou et al. (1997) determined the absolute parameters and the geometrical elements by applying the Wood's model. Edalati and Atighi (1997) compared some parameters of their solution with previous works and confirmed that the system's geometrical configuration is a reverse Algol. Oh et al. (2000) discussed that the system is at poor thermal contact phase of the thermal relaxation oscillation. Recently, Zabihinpoor et al. (2006) analyzed the light curve and suggested new observations to uncover the discrepancy between the suggested geometric shape and the orbital period variation.

The shapes of the radial velocity curves of the system are controversial and no reliable spectroscopic mass ratio exist in the literature. In this study, therefore, we started to the solution by searching the appropriate photometric mass ratio. $q$ values between 0.25 and 0.65 are investigated on the $V$ light curve by increasing the value by 0.05 . We reached the minimum residual when $q=0.45$ which is then taken as an initial value for our simultaneous solution. The uncertainties of the spectral types also required to search for a suitable temperature for the primary component using the light curve. $T_{\mathrm{h}}=10350 \mathrm{~K}$ turned out to be a suitable mean temperature of the hot component and has been used in other studies. Simultaneous solutions obtained with PHOEBE (Prs a and Zwitter, 2005), which uses the WD code (Wilson and Devinney, 1971), was applied to our observations (476 points in $B$ and $V$, and 474 in $R$ ). The gravity darkening coefficients $g_{1}$ and $g_{2}$ are obtained from von Zeipel (1924), Lucy (1967). The albedos $A_{1}$ and $A_{2}$ are adopted from Rucinski (1969). The logarithmic limb-darkening law is used with coefficients adopted from van Hamme (1993) for a solar composition (Table 2). The adjustable parameters are orbital inclination $i$, temperature of secondary component $T_{2}$, surface potential of secondary component $\Omega_{2}$, luminosity $L_{1}$, and mass ratio $q$. The analysis results are summarized in
Table 5

Available light curves data. Phases are given for the light curves LC3, LC5, and LC15 since JDs are not provided. All data for 9 data sets can be found electronically at CDS.

\begin{tabular}{llll}
\hline Data set & Filter & JD/Phase & Magnitude \\
\hline LC6 & B & 2436782.3973 & 0.156 \\
LC6 & B & 2436782.4034 & 0.158 \\
LC6 & B & 2436782.4117 & 0.173 \\
LC6 & B & 2436782.4184 & 0.184 \\
LC6 & B & 2436782.4198 & 0.187 \\
$\vdots$ & $\vdots$ & $\vdots$ & $\vdots$ \\
\hline
\end{tabular}

Table 2. The computed light curves are shown with solid lines in Fig. 1.

All available light curves of the system between 1936-2007 are also collected from the literature and analysed separately (Table 3 ). These light curves are solved by using the initial values that are determined in this study. In addition, light contribution of the third body is set as a fixed parameter since no variation is expected in a period of eighty years. The results are shown in Fig. 3 and listed in Table 4. All available data are provided in Table 5.

Some light curves (LC8, LC12, LC15) show slight asymmetry in the secondary minimum while it is not detected in the others (LC3, LC7, LC14). In this study we also investigate any evidence of a magnetic activity as it was discussed in earlier studies (Hall and Louth, 1990). Either physical structure of the stars or the shape of the light curves did not let us to analyse the curves with spotted model assumption. Some light curves (LC8, LC15), however, can be represented theoretically by a hot surface on the cooler companion. The presence of a hot region, that may be attributed to a mass transfer other than a magnetic activity, could not be proved with the long-term data.

\section{Discussion and Conclusion}

Long term photometric light and period variation of the close binary system GO Cyg are studied in detail. The physical parameters we have determined and listed in Table 6. Because of poor quality data in the previous studies the $\mathrm{O}-\mathrm{C}$ curve was inferred

Table 4

The photometric parameters and $1 \sigma$ errors obtained from the solution of all available light curves. See text for details.

\begin{tabular}{|c|c|c|c|c|c|}
\hline Parameter & LC3 & LC4 & LC5 & LC6 & LC7 \\
\hline$i\left(^{\circ}\right)$ & $75.2(1.5)$ & $77.1(3)$ & 73.42(1.03) & $75.57(8)$ & 76.61(3) \\
\hline$q$ & $0.371(26)$ & $0.435(5)$ & $0.443(17)$ & $0.425(2)$ & $0.457(1)$ \\
\hline$T_{1}(\mathrm{~K})$ & 10350 & 10350 & 10350 & 10350 & 10350 \\
\hline$T_{2}(\mathrm{~K})$ & $6111(240)$ & $6667(64)$ & $6516(144)$ & $6721(30)$ & $6743(18)$ \\
\hline$\Omega_{1}$ & 2.616 & 2.764 & 2.749 & 2.729 & 2.786 \\
\hline$\Omega_{2}$ & $2.603(65)$ & $2.722(18)$ & $2.627(18)$ & $2.662(3)$ & $2.702(2)$ \\
\hline$\left(\frac{L_{1}}{L_{1}+L_{2}}\right)_{B}$ & - & $0.934(47)$ & $0.932(101)$ & $0.927(18)$ & $0.913(13)$ \\
\hline V & $0.938(124)$ & $0.896(53)$ & $0.895(122)$ & $0.892(21)$ & $0.877(14)$ \\
\hline$R$ & - & - & - & - & - \\
\hline$r_{1}$ & $0.4676(65)$ & $0.4512(11)$ & $0.4528(34)$ & $0.4549(3)$ & $0.4490(2)$ \\
\hline \multirow[t]{2}{*}{$r_{2}$} & $0.2965(268)$ & $0.3184(46)$ & $0.3420(151)$ & $0.3238(16)$ & $0.3352(13)$ \\
\hline & LC8 & LC14 & LC15 & LC16 & \\
\hline$i\left(^{\circ}\right)$ & $77.02(2)$ & $74.2(1)$ & $74.5(1)$ & $75.67(3)$ & \\
\hline$q$ & $0.424(2)$ & $0.457(2)$ & $0.461(2)$ & $0.428(9)$ & \\
\hline$T_{1}(\mathrm{~K})$ & 10350 & 10350 & 10350 & 10350 & \\
\hline$T_{2}(\mathrm{~K})$ & $6688(23)$ & $6651(50)$ & $6709(35)$ & $6478(262)$ & \\
\hline$\Omega_{1}$ & 2.713 & 2.785 & 2.804 & 2.735 & \\
\hline$\Omega_{2}$ & $2.711(6)$ & $2.674(3)$ & $2.612(3)$ & $2.686(4)$ & \\
\hline$\left(\frac{L_{1}}{L_{1}+L_{2}}\right)_{B}$ & $0.913(13)$ & $0.933(15)$ & $0.922(32)$ & 0.941() 59 & \\
\hline V & $0.906(16)$ & $0.885(36)$ & $0.862(30)$ & $0.908(48)$ & \\
\hline$R$ & - & - & - & $0.875(46)$ & \\
\hline$r_{1}$ & $0.4567(4)$ & $0.4490(3)$ & $0.4472(3)$ & $0.4542(2)$ & \\
\hline$r_{2}$ & $0.3110(21)$ & $0.3405(15)$ & $0.3680(22)$ & $0.3189(12)$ & \\
\hline
\end{tabular}


Table 6

Absolute parameters of GO Cyg. The standard errors $1 \sigma$ in the last digit are given in parentheses.

\begin{tabular}{llll}
\hline Parameter & Unit & Pr. & Sec. \\
\hline Mass (M) & $M_{\odot}$ & $3.0(2)$ & $1.3(1)$ \\
Radius (R) & $R_{\odot}$ & $2.50(12)$ & $1.75(9)$ \\
Temperature $\left(T_{\text {eff }}\right)$ & $\mathrm{K}$ & 10350 & 6490 \\
Luminosity $(\mathrm{L})$ & $L_{\odot}$ & $64(9)$ & $4.9(7)$ \\
Absolute bolometric magnitude $\left(M_{b}\right)$ & $\mathrm{mag}$ & 0.23 & 3.03 \\
Period change rate $(\dot{P})$ & $\mathrm{d} / \mathrm{yr}$ & $-1.4 \times 10^{-9}$ & \\
Mass transfer ratio $(\dot{M})$ & $M_{\odot} / \mathrm{yr}$ & $1.5 \times 10^{-9}$ & \\
Seperation between stars $(a)$ & $R_{\odot}$ & $5.5(3)$ & \\
\hline
\end{tabular}

to be parabolic one. In this study with our data we show that the system has a third body with a $92.3 \mathrm{yr}$ orbital period. Since the primary component filled its Roche lobe we searched for a clue for the mass transfer by a technique other than the $O-C$ analysis. Separate solutions of six available light curves starting from 1950 to present indicate a period decrease. The period change variation vs. years shows a downward parabola. This can be considered as a mass transfer from the more massive companion to the less massive one. This solution yields the amount of the period decrease as $-1.4 \times 10^{-9} \mathrm{~d} / \mathrm{yr}$ with a mass transfer rate of $1.5 \times 10^{-9} M_{\odot} / \mathrm{yr}$. In addition, the period of this parabolic variation is too long to detect in the $\mathrm{O}-\mathrm{C}$ curve constructed with the available data.

The system has been studied spectroscopically but no accurate radial velocity study of it is available in the literature. In systems like GO Cyg the luminous primary star makes it difficult to treat the system as a double-lined binary. Determination of the physical parameters requires the knowledge of accurate mass ratio and the mass of the primary component. The best result obtained by a $q$-search technique in the light curve analysis. In what follows solution to allowed the mass ratio to vary allowing with other parameters when the solve for an orbital the light curves. Our solutions all have similar results for all nine light curves. Our results are given in Tables 2 and 3. Spectral studies of NCB systems (e.g. GO Cyg) are quite difficult because of their nature. The mass of the primary, therefore, estimated according to their colors, spectral types etc.. In this study, we used recently published astrophysical data of well known stars (e.g., Torres et al., 2010; Yakut and Eggleton, 2005; Drilling and Landolt, 2000) to estimate the primary's (the massive and lumonious one) mass. By studying stars with similar luminosities and spectral types, the mass of the primary is assumed to be $3.0 M_{\odot}$. This is also consistent with the values given in the literature. The physical parameters of the system is given in Table 6 , the results are consistent with the similar systems on the

Table 7

Physical parameters of some well known massive $\left(M>1.6 M_{\odot}\right)$ NCB systems. The data is taken from Yakut and Eggleton (2005) except RZ Dra (Erdem et al., 2011), RU UM (Lee et al., 2008), GW Gem (Lee et al., 2009), EE Aqr (Wronka et al., 2010), KQ Gem (Zhang, 2010).

\begin{tabular}{llllllr}
\hline Name & Sp.T & $\mathrm{P}(\mathrm{d})$ & $M_{1}$ & $\mathrm{M}_{2}$ & $\log L_{1}$ & \multicolumn{1}{c}{$\log L_{2}$} \\
\hline RZ Dra & A5 & 0.5509 & 1.63 & 0.70 & 0.988 & -0.131 \\
RT Scl & F0 & 0.5116 & 1.63 & 0.71 & 0.733 & -0.310 \\
RV Crv & F3 & 0.7473 & 1.64 & 0.45 & 0.907 & -0.060 \\
AG Vir & A8 & 0.6427 & 1.67 & 0.53 & 1.021 & 0.262 \\
DO Cas & A7 & 0.6847 & 1.70 & 0.53 & 1.124 & -0.469 \\
KQ Gem & F5 & 0.4080 & 1.70 & 0.40 & 1.199 & -0.319 \\
SW Lyn & F2 & 0.6441 & 1.72 & 0.90 & 0.808 & -0.208 \\
GW Gem & A7 & 0.6594 & 1.74 & 0.80 & 0.985 & 0.100 \\
FO Vir & A8 & 0.7756 & 1.75 & 0.27 & 1.199 & -0.319 \\
YY Cet & A8 & 0.7905 & 1.84 & 0.94 & 1.093 & 0.279 \\
RS Ind & A9 & 0.6241 & 2.00 & 0.62 & 0.984 & -0.229 \\
V836 Cyg & B9.5 & 0.6534 & 2.20 & 0.78 & 1.491 & 0.127 \\
EE Aqr & A9.5 & 0.5090 & 2.24 & 0.72 & 0.897 & -0.378 \\
RU UMi & A9 & 0.5249 & 2.32 & 0.76 & 0.883 & -0.268 \\
GO Cyg & B8 & 0.7178 & 3.00 & 1.30 & 1.806 & 0.690 \\
\hline
\end{tabular}

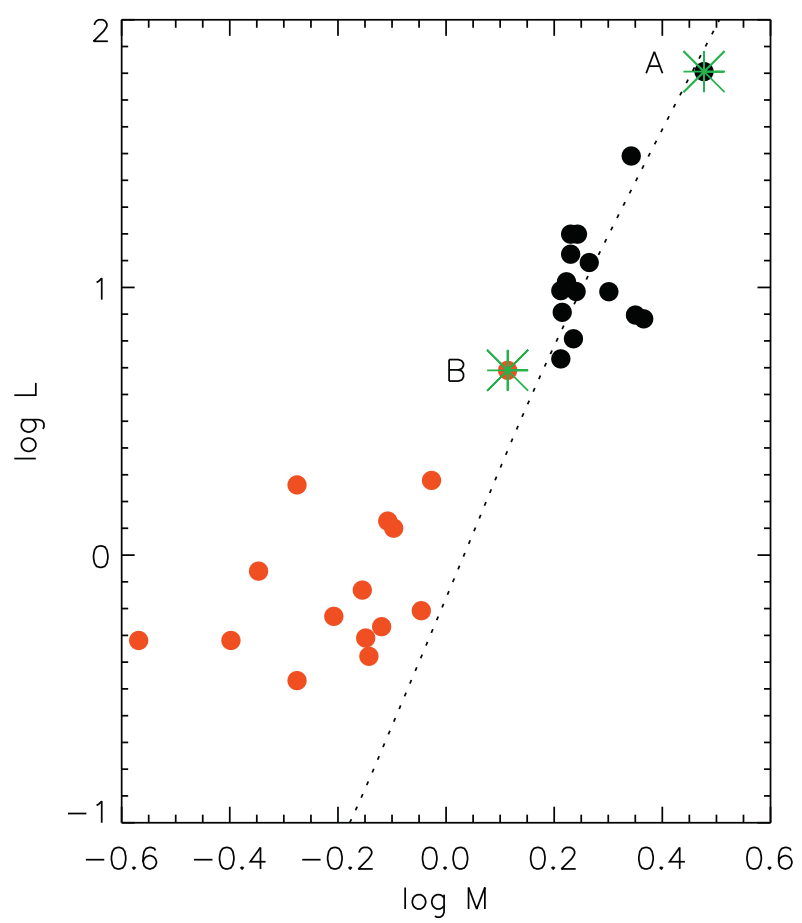

Fig. 4. Plot of the $M-L$ plane of the some NCB systems. The ZAMS line is taken from Pols et al. (1995).

$M-R, M-L$ and the Hertzsprung-Russell diagrams given by Yakut and Eggleton (2005). Our results shows that GO Cyg has the most massive components among the known NCB systems. We collected physical parameters of the NCB systems whose primary components are relatively massive (Table 7). Mass-luminosity diagram of binaries listed in Table 7 is shown in Fig. 4. The locations of GO Cyg A and B in the $M-L$ diagram are consistent with the other NCB systems.

Observational results of semi-detached systems show that while in some cases the primary component fills its Roche lobe (GO Cyg) in other cases the secondary fills its Roche lobe (V836 Cyg, Yakut et al., 2005). These differences are a sign for the evolutionary stage of the binary system (for details we refer Yakut and Eggleton, 2005; Eggleton, 2006; Eggleton, 2010). The orbital, geometrical, and physical parameters of GO Cyg presented in this study indicate the Roche lobe filling star is the primary (the massive and the hotter one). We show that the primary may even transfer mass with at a low rate. Contrary to the very low mass stars, mass loss rate due to the magnetic stellar winds can be expected since the convective layer is small in the systems with intermediate/low mass components (e.g. GO Cyg). The results indicate the system GO Cyg evolves under the proximity effect, low rate mass transfer between the components $\left(\dot{M}=1.5 \times 10^{-9} M_{\odot} /\right.$ $\mathrm{yr})$ and the third body can also remove angular momentum from the binary orbit.

\section{Acknowledgments}

This study was supported by the Turkish Scientific and Research Council (TÜBITAK 109T047 and 111T270) and Ege University Research Fund. KY+VK acknowledges support by the Turkish Academy of Sciences (TÜBA). We thank to an anonymous referee, J.J. Eldridge and E.R. Pekünlü for their valuable comments and suggestions.

\section{References}

Cester, B., Giuricin, G., Mardirossian, F., Mezzetti, M., 1979. Acta Astron. 29, 433. Chochol, D. et al., 2006. Ap\&SS 304, 93. 
Drilling, J.S., Landolt, A.U., 2000. Allen's Astrophys. Quant., 381.

Edalati, M.T., Atighi, M., 1997. Ap\&SS 253, 107.

Eggleton, P., 2006. Evolutionary Processes in Binary and Multiple Stars, Cambridge Astrophysics Series No. 40.

Eggleton, P.P., 2010. NewAR 54, 45.

Elkhateeb, M.M., 2005. JKAS 38, 13.

Erdem, A., Zola, S., Winiarski, M., 2011. NewA 16, 6.

Erkan, N., Erdem, A., Akın, T., Aliçavuş, F., Soydugan, F., 2010. IBVS 5924, 1.

Hall, D.S., Louth, H., 1990. JApA 11, 271.

Irwin, J.B., 1959. AJ 64, 149.

Jassur, D.M.Z., 1997. Ap\&SS 249, 111.

Jones, R.A., Snyder, L., Frey, G., Dalmau, FJ., Aloy, J., Bonvehi, L., 1994. IAPPP 54, 34

Kalomeni, B., Yakut, K., Keskin, V., Değirmenci, Ö.L., Ulaş, B., Köse, O., 2007. AJ 134, 642.

Köse, O., Kalomeni, B., Keskin, V., Ulaş, B., Yakut, K., 2011. AN 332, 626.

Kreiner, J.M., Kim, C.-H., Nha, I.-S., 2001, aocd.book.

Lee, J.W., Kim, C.-H., Kim, S.-L., Lee, C.-U., Han, W., Koch, R.H., 2008. PASP 120, 720

Lee, J.W., Kim, S.-L., Lee, C.-U., Kim, H.-I., Park, J.-H., Park, S.-R., Koch, R.H., 2009. PASP 121, 104.

Liau, S.P., 1935. POLyo, 1

Lucy, L.B., 1967. Z. Astrophys. 65, 89.

Mannino, G., 1963. MmSAI 34, 191.

Oh, K.-D., Kang, Y.W., Ra, K.S., Park, H.S., 2000. Ap\&SS 271, 303.

Oprescu, G., Dumitrescu, A., Suran, M.D., Rovithis, P., Rovithis-Livaniou, H., 1996. RoAJ 6, 119.

Ovenden, M.W., 1954. MNRAS 114, 569.
Payne-Gaposchkin, C., 1935. BHarO 898, 3.

Pearce, J.A., 1933. JRASC 27, 62.

Pierce, N.L., 1939. AJ 48, 113.

Pols, O.R., Tout, C.A., Eggleton, P.P., Han, Z., 1995. MNRAS 274, 964.

Popper, D.M., 1957. APJS 3, 107.

Pribulla, T. et al., 2009. AJ 137, 3655.

Prs a, A., Zwitter, T., 2005. ApJ 628, 426.

Rovithis, P., Rovithis-Livaniou, H., Niarchos, P.G., 1990. A\&AS 83, 41.

Rovithis-Livaniou, H., Rovithis, P., Oprescu, G., Dumitrescu, A., Suran, M.D., 1997. A\&A 327, 1017

Rucinski, S.M., 1969. Acta Astronom. 19, 245.

Schneller, H., 1928. AN 235, 85.

Sezer, C., Gülmen, O., Güdür, N., 1985. IBVS 2743, 1.

Sezer, C., Gülmen, O., Güdür, N., 1993. Ap\&SS 203, 121.

Torres, G., Andersen, J., Giménez, A., 2010. A\&ARv 18, 67.

Ulas, B., Kalomeni, B., Keskin, V., Kose, O., Yakut, K., 2011, <arxiv:1107.0277>

van Hamme, W., 1993. AJ 106, 2096.

von Zeipel, H., 1924. MNRAS 84, 665

Vukasović, M., 1997. IAPPP 67, 11.

Wilson, R.E., Devinney, E.J., 1971. ApJ 166, 605.

Wronka, M.D., Gold, C., Sowell, J.R., Williamon, R.M., 2010. AJ 139, 1486

Yakut, K., Eggleton, P.P., 2005. ApJ 629, 1055.

Yakut, K., Ulaș, B., Kalomeni, B., Gülmen, Ö., 2005. MNRAS 363, 1272.

Zabihinpoor, S.M., Dariush, A., Riazi, N., 2006. Ap\&SS 302, 27.

Zhang, L.-Y., 2010. PASP 122, 309. 